\title{
Borderline personality disorder: an update for neurologists
}

\author{
Katherine Hall, ${ }^{1}$ Paul Moran ${ }^{2}$
}

'Bristol Medical School, University of Bristol, Bristol, UK ${ }^{2}$ Centre for Academic Mental Health, Population Health Sciences, Bristol Medical School, University of Bristol, Bristol, UK

Correspondence to Dr Katherine Hall, Bristol Medical School, University of Bristol, Bristol BS8 2BN, UK; kh9743@my.bristol.ac.uk

Accepted 31 May 2019 Published Online First 27 June 2019

\section{Check for updates}

(c) Author(s) (or their employer(s)) 2019. No commercial re-use. See rights and permissions. Published by BMJ.

To cite: Hall K, Moran P. Pract Neurol 2019;19:483491.

\begin{abstract}
The value and challenges of establishing and maintaining a successful doctor-patient relationship are thrown into sharp relief in the treatment of people with borderline personality disorder. We present an overview of this common and important condition, its epidemiology, cause, common comorbidities and neurological associations. We then propose a practical, psychologically informed framework for enhancing the therapeutic alliance for the jobbing neurologist in managing these patients.
\end{abstract}

\section{INTRODUCTION}

Neurologists encounter people with personality disorder in their outpatient clinics, the emergency department and on the medical wards. People with borderline personality disorder (BPD) characteristically experience distressing disturbances in self-image, impulsivity, problems with emotional regulation and pervasive difficulties in interpersonal relationships. They are at increased risk of self-harm and suicide, and experience higher rates of somatisation. Reasons for neurology referrals in this patient group include dissociative seizures, conversion disorders and other functional neurological impairments, of course in addition to standard neurological presentations, including those occurring in the context of head injury, as well as alcohol and substance use.

In such contexts, successful management of the prima facie problem is likely to involve a deeper consideration of a person's life experiences and subsequent relational difficulties; these may be contributing to their neurological presentation and will certainly impact on the doctor-patient relationship. Although it is not a neurologist's remit to treat underlying personality disorder, an astute approach to the therapeutic alliance will enhance treatment outcomes in these patients. In order to achieve this, the neurologist will benefit from an understanding of this common condition and of associated pitfalls frequently encountered by health professionals in the approach to management.

\section{DEFINITION 'Borderland'}

The term 'borderline personality', now a source of confusion, was first used in 1938 by Adolph Stern to describe a group of patients who 'fit frankly neither into the psychotic nor into the psychoneurotic group', but who inhabited a realm between the two. ${ }^{1}$

The definition and classification of BPD have been heavily debated, reflecting persisting uncertainty in how best to conceptualise the underlying psychopathology. Personality disorder is defined in the International Classification of Diseases (ICD) - 11 as:

"a relatively enduring and pervasive disturbance in how individuals experience and interpret themselves, others, and the world that results in maladaptive patterns of cognition, emotional experience, emotional expression, and behaviour". ${ }^{2}$

These maladaptive patterns commonly have their first manifestations in childhood and adolescence, and are typically associated with instability in interpersonal relationships. In ICD-10, 'borderline type' personality disorder comes under the umbrella term of 'emotionally unstable personality disorder', alongside 'impulsive type' personality disorder. As there is considerable overlap between the categories, the term 'emotionally unstable personality disorder' is sometimes used interchangeably with BPD in the clinical setting. The move away from categorical descriptions of personality disorder towards dimensional classification in ICD-11 signifies a major nosological overhaul. ${ }^{3}$ Box 1 shows the DSM-5 criteria for $\mathrm{BPD}$; five or more of these criteria are required to make the diagnosis. ${ }^{4}$ 
Box 1 Features of borderline personality disorder

Frantic efforts to avoid real or imagined abandonment.

- A pattern of unstable and intense interpersonal relationships, typically characterised by alternating between extremes of idealisation and devaluation.

- Identity disturbance, manifested in markedly and persistently unstable self-image or sense of self.

- Impulsivity manifested in potentially self-damaging behaviours (eg, risky sexual behaviour, reckless driving, excessive alcohol or substance use, binge eating).

- Recurrent episodes of self-harm including suicide attempts.

- Emotional instability due to marked reactivity of mood. Fluctuations of mood may be triggered either internally (by one's own thoughts) or by external events. As a consequence, the individual experiences intense dysphoric mood states, which typically last for a few hours but may last for up to several days.

- Chronic feelings of emptiness.

- Difficulty controlling anger.

- Transient dissociative symptoms or psychotic-like features such as transient hallucinations and paranoia, typically in situations of high affective arousal.

Adapted from the ICD-11 Clinical Descriptions and Diagnostic Guidelines for Personality Disorder, ${ }^{49}$ based on the American Psychiatric Association's Diagnostic and Statistical Manual of Mental Disorders (DSM) $-5 .{ }^{4}$

\section{What's in a name?}

It is noteworthy that the term 'personality disorder' is all too often used in a pejorative sense, with authors of a 2015 Lancet series on the subject going so far as to lament that 'it has now become more a term of abuse than a diagnosis'. ${ }^{2}$ It is important for all doctors to maintain an open mind and compassionate approach when dealing with patients diagnosed with personality disorder. This requires skill and patience as the psychopathology of these patients usually chiefly manifests itself in the manner in which they interact with others.

\section{EPIDEMIOLOGY}

In the Western world, where most of the research in this area has been conducted, BPD has a lifetime prevalence of around $6 \%{ }^{5}$ and a community prevalence of approximately 1\%-3\%. ${ }^{5}$ Sociodemographic correlates include younger age, living in urban centres and not living with a partner, ${ }^{7}$ as well as low socioeconomic status. ${ }^{8}$

Although women with BPD are disproportionately represented in clinical settings compared with men, community samples do not identify a clear difference in its prevalence between women and men. ${ }^{179}$

BPD affects $4 \%-6 \%$ of those attending primary care services, ${ }^{1011}$ and people with BPD attend their general practitioner more frequently and report greater psychosocial impairment ${ }^{12}$ than those without BPD. As many as $40 \%$ of those in contact with secondary mental health services are affected by personality disorder, of which BPD is the most common type. ${ }^{13}$

\section{CAUSE}

In common with other psychiatric disorders, the aetiology of BPD is multifactorial. Various interdisciplinary attempts have been made to integrate the complex array of underlying aetiological factors, yet there remains much to be discovered. One such influential model is Linehan's biosocial model, which proposes BPD as arising from an emotionally vulnerable temperament (biological vulnerabilities) interacting with an invalidating environment (psychosocial factors). ${ }^{14}$

Recognised contributors to BPD include genetic vulnerabilities and a multitude of environmental factors, particularly adverse childhood experiences. ${ }^{7}$

\section{Genetics}

Twin studies have shown moderate to high heritability for BPD. ${ }^{15}$ No robust evidence has yet been established for single-candidate genes, and genome-wide association studies in this area are in their relative infancy. ${ }^{16}$

${ }^{17}$ Several small gene-environment studies have identified interactions which need verifying in larger, prospective cohorts. ${ }^{17}$

Epigenetic studies examine how environmental conditions affect gene expression and are increasingly being considered a promising avenue for exploring the cause of personality disorders. ${ }^{18}$ Several small studies suggest epigenetic modifications in people with BPD in genes related to stress and neurodevelopment, with environmental factors such as childhood trauma having a measurable impact on genes responsible for neurofunctioning. ${ }^{17} 18$ For example, people with BPD have significantly higher DNA methylation, that is, evidence of epigenetic modification, of the brain-derived neurotrophic factor gene, which is involved in neurodevelopment. ${ }^{19}$ Such epigenetic changes may pave the way for new treatment approaches, but further research is needed to establish this. ${ }^{19}$

\section{Psychosocial factors}

Early environmental and familial risk factors for BPD have been consistently demonstrated over the years. These include childhood trauma such as neglect and abuse (emotional, physical or sexual), familial adversity, a history of psychopathology in the caregiver, as well as parental emotional under-involvement. ${ }^{18}$

The marked association between childhood sexual abuse and the development of BPD, and the positive correlation between the severity of BPD and the severity of the abuse experienced, have led some researchers to argue that people with BPD might be better thought of as having a chronic form posttraumatic stress disorder. ${ }^{20}$ Clearly not all people who 
experience abuse go on to develop BPD, and the fact that abuse alone is neither necessary nor sufficient for its development points to other predisposing (such as genetic) and contextual mediating factors. ${ }^{1}$ For example, emotional denial of a child's experience of trauma by their caregiver has been identified as a predictor for BPD. ${ }^{21}$ The family and wider social environment are considered to play a critical role, as well as whether a child's perspective on their emotional world is perceived to be validated. ${ }^{1}$

\section{Note on attachment}

Attachment theory, developed by John Bowlby, posits the universal human need for close affectional bonds as a prerequisite for normal development.

When a child has a mental representation of the caregiver as being available and responsive to their needs, secure attachment occurs. BPD is strongly associated with insecure attachment, ${ }^{1}$ which occurs when selfperceptions of internal states are instead undermined. It is not clear to what extent attachment difficulties in BPD are causal to or result from the emotional disturbance and impulsivity associated with the condition.

\section{Neurobiology}

Several neural correlates associated with BPD have been reported. Structurally, reduced amygdala and hippocampal volumes have been observed. ${ }^{1}$ Functionally, evidence exists for increased limbic activity and decreased prefrontal activity in patients with BPD in response to emotional stimuli, in keeping with clinical observations of emotional dysregulation. ${ }^{22}$

People with BPD have also been consistently shown to experience a negative response bias to ambiguous or neutral facial expressions, as well as an enhanced sensitivity to fearful expressions. ${ }^{23}$ Contrary to popular misconception, people with BPD have demonstrable accuracy in determining mental states in response to complex social stimuli, ${ }^{23}$ and these patients are exquisitely sensitive to the states of mind of those around them.

On examination, patients with BPD have been found to exhibit higher rates of neurological soft signs in keeping with underlying non-focal neurological dysfunction. ${ }^{24}$

\section{COMORBIDITIES}

\section{Psychiatric comorbidities}

Depression, anxiety and substance use disorders, as well as eating disorders, post-traumatic stress disorder and bipolar disorder, are all important diagnostic comorbidities. ${ }^{115}$

It can be challenging for the clinician to distinguish comorbidity in patients with BPD; for example, the emotional dysregulation seen in people with BPD may mimic depression, mania or anxiety. In this respect, it is invariably helpful to obtain an informant account in order to establish the duration and onset of symptoms.
Many people with BPD engage in repeated episodes of self-harm and endure recurrent thoughts of suicide and suicide attempts; the mortality rate for people with BPD from suicide has been estimated to be as high as $8 \%-10 \%, 50$ times higher than that in the general population. ${ }^{15}$

\section{Physical comorbidities}

People with personality disorders have an average life expectancy starkly reduced by around two decades compared with the general population. ${ }^{25} 26$ The excess mortality cannot be attributed to increased suicide rates alone and it is now apparent that people with BPD experience significantly poorer physical health compared with individuals not diagnosed with BPD. Rates of cardiovascular and cerebrovascular disease are substantially increased, ${ }^{24}$ and associations have also been reported between BPD and gastrointestinal and hepatic diseases, arthritis, sleep disorders and chronic pain, as well as syncope and seizures. ${ }^{27}$ The underlying mechanisms for this disproportionate burden of illness are unknown; lifestyle factors such as higher rates of smoking and poorer diet are likely to be implicated, as well as the toll taken by chronic levels of psychological stress on the body. ${ }^{27}$

People with BPD may also present to neurologists given their increased rates of migraine-related disability and a lower likelihood of responding to pharmacological headache treatment compared with controls, ${ }^{28}$ or due to neurological sequelae of selfinjury and substance use disorders.

\section{Functional neurological associations}

Across the boundary between psychiatric and physical comorbidities of BPD lie the somatoform disorders, renamed 'somatic symptom disorder' in DSM-5 and 'bodily distress disorder' in ICD-11. ${ }^{29}$

Under this category is 'conversion' (functional neurological symptom) disorder, which refers to patients with neurological symptoms such as seizures, limb weakness and movement disorders without identifiable organic disease. ${ }^{30}$ Conversion disorder is one of the most common reasons for referral to neurology, ${ }^{30}$ and co-occurring BPD has been found to affect a significant proportion of people with conversion disorder $(22 \%){ }^{31}$

Substantial comorbidity has been demonstrated between BPD and somatic symptom disorders, and these conditions also share two important commonalities: an association with a history of childhood trauma and features of emotional dysregulation. ${ }^{31}$ Such common denominators have also been noted between BPD and the specific example of dissociative (nonepileptic) seizures, which can fall within both conversion and dissociative disorders. ${ }^{32}$

\section{Functional neurological symptom disorder}

For much of the last century since Breuer and Freud's hypothesis that psychological distress was 'converted' 
into physical symptoms, psychiatric formulations of the so-named disorder have dominated.

The nosological shift from 'conversion' to 'functional neurological symptom' disorder in DSM-5 represents an important swing of the pendulum back towards neurology, with both fields having valuable perspectives. To neurologists, functional neurological symptom disorders are no longer diagnoses to be excluded and referred back. Rather, neurologists are increasingly recognised as having an important therapeutic role. ${ }^{33}$ Reflecting this shift, diagnostic criteria now demand positive neurological examination features (eg, Hoover's sign of functional leg weakness). ${ }^{34}$

\section{Diagnostic overshadowing}

In closing this section about neurological associations of $\mathrm{BPD}$, we think that it is important to remind the reader of this process where the cause of physical symptoms is misattributed to mental illness, a process termed 'diagnostic overshadowing'. Examples include people with psychiatric disorders and ischaemic heart disease being less likely to receive the required revascularisation procedure than those without mental illness, and hospital admission for diabetic complications being less likely to be offered to those with comorbid psychiatric disorders than those without. ${ }^{34}$

A range of contributors to this phenomenon have been postulated, including clinicians' biases, naivety about how psychiatric disorders present, and complexities of doctor-patient communication. ${ }^{35}$

Patients will therefore benefit from neurologists retaining their sharp clinical eye for organic neurological diseases regardless of the thickness of the clinical notes, psychiatric history or current affective presentation.

\section{SUMMARY OF PSYCHIATRIC MANAGEMENT OF BPD}

Management options for BPD have considerably advanced over the past 20 years. The primary treatment is considered to be psychotherapy, of which several methods have been adapted for the disorder. These include dialectical behavioural therapy, cognitive-behavioural therapy, schema-focused therapy and specific forms of psychodynamic psychotherapy such as mentalisation-based therapy and transference-focused psychotherapy. ${ }^{36}$ There is some evidence that these approaches are more effective than treatment as usual in certain aspects of BPD such as suicidality. ${ }^{163738}$ Arts therapies and family therapy can also play important roles. ${ }^{1}$

Pharmacotherapy for BPD is only advised as adjunctive treatment, either for comorbidities or for shortterm symptomatic relief during crises. ${ }^{1}$ Guidelines from the National Institute for Health and Care Excellence (NICE) are clear that:

"drug treatment should not be used specifically for borderline personality disorder or for the individual symptoms or behaviour associated with the disorder (for example, repeated self-harm, marked emotional instability, risk-taking behaviour and transient psychotic symptoms)".,38

During a crisis, short-term pharmacological treatment can help when the decision is reached collaboratively with the patient. Where possible, a single drug with low addictive properties and relative safety in overdose is preferred, for example, a sedative antihistamine. For example, promethazine can be used at a dose of $25 \mathrm{mg}$ to $50 \mathrm{mg}$, with a maximum dose within 24 hours of $100 \mathrm{mg}$, though its use is off-license. NICE recommends using the minimum effective dose of any medication, and developing a plan to stop treatment started during the crisis, usually within 1 week. ${ }^{38}$

\section{Risk management}

Many patients with BPD self-harm, for example, by cutting, scratching or burning. Such behaviour can serve many different functions, from dampening down painful emotions to attempting to reconnect with one's emotions in the face of dissociation or feelings of emptiness, or as a way of regulating relationships by either pulling people in as rescuers or pushing them away. ${ }^{39}$ They may also suffer from chronic thoughts of suicide. However, it is important to consider obtaining urgent advice from a psychiatrist if there has been a recent change in the patient's self-harming (eg, escalation now requiring medical attention) or when a patient states an intensified intent to end their life in relation to their suicidal thinking.

\section{Some therapies de-jargonised}

Dialectical behaviour therapy

- Combines certain principles of cognitive-behavioural therapy with mindfulness, Zen-Buddhistic and dialectical thinking strategies. ${ }^{36}$

- Aims for behavioural change and enhanced distress tolerance, emotional regulation and interpersonal behaviour.

Mentalisation-based therapy

- Draws on psychodynamic and attachment-based principles.

- Aims to improve a person's ability to apprehend their own feelings and those which they evoke in others.

Schema-focused therapy

- Combines cognitive-behavioural therapy with psychoanalysis.

- Aims to identify core maladaptive themes stemming from unmet childhood emotional needs, encouraging more adaptive coping styles.

Transference-focused psychotherapy

- Employs clarification, confrontation and interpretation of the therapeutic relationship to work towards integrated representations of self and others. 


\section{Can people with BPD recover?}

Prospective longitudinal research has shown that the majority of patients with BPD experience a significant remission in symptoms (notably self-harm) over a 10-year period. However, functional impairment remains an enduring feature of BPD and many people with BPD experience lifelong difficulties in maintaining work and intimate relationships. ${ }^{40} 41$

\section{KEY CONSIDERATIONS FOR THE DOCTOR- PATIENT RELATIONSHIP IN THE CONTEXT OF PATIENTS WITH BPD}

In this section, we outline some ideas and opinions derived through our own experience, supplemented by research relevant to neurological practice.

\section{Recognising its importance}

It is well known that a robust and positive doctorpatient relationship is associated with better treatment adherence and outcomes, and that a poor relationship is linked to higher dropout rates from treatment. ${ }^{42}$

In a previous review for this journal, Stone compellingly outlined how, with a good therapeutic alliance, a neurological assessment itself can also be a form of treatment for functional disorders. Useful tips included emphasising that the symptoms are real and potentially treatable, outlining the positive nature of the diagnosis (rather than treating it as a diagnosis of exclusion), simple signposting to appropriate help and information possibly including psychological services, and offering outpatient follow-up. ${ }^{33}$

In the context of psychotherapy, the trust and empathy developed within therapeutic relationships is believed to contribute to a corrective relational experience in these patients who might otherwise have had very little reason to trust in others. ${ }^{15}$ These principles can also be usefully applied in the management of organic neurological disorders in people with comorbid BPD.

\section{Collaboration}

Whatever the reason for referral, inviting and encouraging the patient's lived experience and understanding of the problem will invariably be a constructive place to start. ${ }^{32}$ In the example of people with BPD and dissociative (non-epileptic) seizures, the building of a shared explanatory framework for the cause of seizures is preferable to the imposition of a medically modelled explanation-with higher chances of patients both understanding and accepting their diagnosis. ${ }^{3243}$ A collaboratively-built, blame-free narrative of the person's difficulties also forms a foundation on which to negotiate realistic treatment goals. ${ }^{29}$

In addition, collaboration within the treating clinical team is of particular importance for these patients. Because people with personality disorder often have disordered attachment styles and difficulties in relationships, powerful dynamics can emerge with and within the medical teams. One example is 'splitting', a

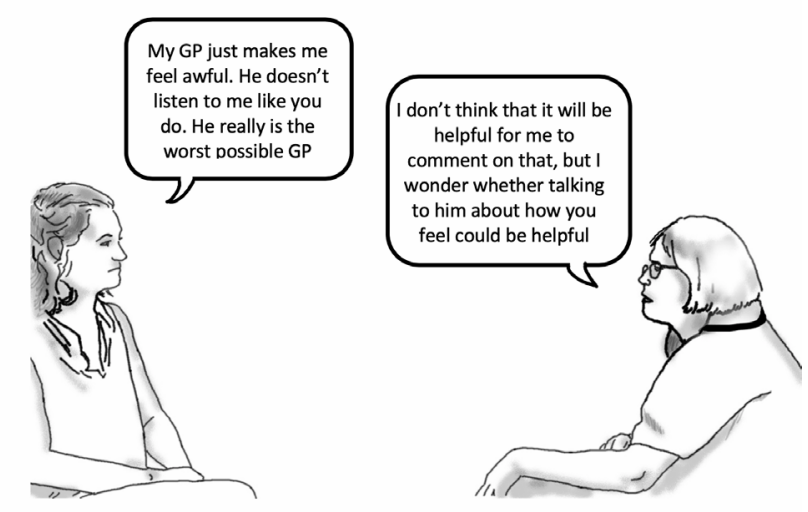

Figure 1 Suggestion on how to notice and avoid being drawn into a conversation possibly around 'splitting'. GP, general practitioner.

suggested unconscious psychological response to stress which leads to experiencing the self or others as either entirely 'good' or entirely 'bad'. It is proposed to originate in early life, for example, through allowing a child to 'split off' threatening perceptions of a caregiver and thereby maintaining a 'good' representation of that caregiver. ${ }^{44}$ This process is thought to translate interpersonally, with patients perceiving certain members of staff as 'good' while others are perceived to be 'bad'. The net result of this is that sometimes staff can be unwittingly drawn into bitter arguments about a patient's behaviour or management. ${ }^{15}$ However, if team members are able to maintain a supportive approach to the patient, this may go some way to mitigating this phenomenon. Moreover, as is done in dialectical behavioural therapy, clinicians confronted with denigration of another healthcare professional by the patient are encouraged neither to agree with the patient nor to defend the other, as shown in figure 1, to avoid becoming trapped in an unhelpful conversation. $^{44}$

A summary of splitting and other patterns, along with suggested solutions, is provided in table 1. Being alert to these manifestations may help with identifying, understanding and assisting patients with personality difficulties. $^{32}$

Service-level collaboration is also critical. Poor communication between psychiatrists and neurologist colleagues has been attributed to worse outcomes for patients with dissociative (non-epileptic) seizures. ${ }^{45}$ Innovative collaborative approaches have been proposed, such as ongoing psychological supervision for neurologists, which could be provided, for example, by a psychiatrist consulting for an epilepsy service. ${ }^{46}$

\section{Being mindful of how a patient's background history can impact their current behaviour}

During difficult consultations, we find it helpful to be cognisant that people act in the way that they do for all sorts of complex reasons; people with personality 
Table 1 Treating people with BPD: some potentially difficult dynamics, underlying explanations and possible solutions

\begin{tabular}{|c|c|c|}
\hline $\begin{array}{l}\text { Examples of perceived behaviour of } \\
\text { people with BPD } 50\end{array}$ & Possible explanations for this behaviour & Possible solutions for the doctor \\
\hline High use of healthcare & $\begin{array}{l}\text { High burden of psychiatric and physical } \\
\text { comorbidities, coupled with health anxiety } \\
\text { and fear of abandonment }\end{array}$ & $\begin{array}{l}\text { Allowing enough time during the consultation to } \\
\text { make the patient feel understood } \\
\text { Avoiding 'bouncing' patients between specialties } \\
\text { where possible }\end{array}$ \\
\hline Apparent intentional sabotage of care & $\begin{array}{l}\text { Part of self-destructive behaviour due to low } \\
\text { self-worth } \\
\text { Fear of abandonment by health professionals }\end{array}$ & $\begin{array}{l}\text { Maintaining an open and validating approach } \\
\text { Organising follow-up }\end{array}$ \\
\hline Difficulty establishing trust & $\begin{array}{l}\text { Histories of being repeatedly let down or } \\
\text { taken advantage of in the past }\end{array}$ & $\begin{array}{l}\text { Working towards a collaborative, transparent } \\
\text { approach with careful explanations } \\
\text { Sticking to appointment times and agreements } \\
\text { Organising follow-up }\end{array}$ \\
\hline Misunderstandings and angry reactions & $\begin{array}{l}\text { Negative interpretative bias to ambiguous } \\
\text { faces in people with BPD }\end{array}$ & $\begin{array}{l}\text { A genuinely compassionate approach from the } \\
\text { physician will often instil greater patient confidence }\end{array}$ \\
\hline 'Splitting' in clinical teams & $\begin{array}{l}\text { Emotional dysregulation in patients can } \\
\text { provoke strong reactions in clinicians which } \\
\text { can become polarised }\end{array}$ & $\begin{array}{l}\text { Recognising the pattern and using it informatively } \\
\text { Effective teamwork and adequate supervision }\end{array}$ \\
\hline
\end{tabular}

BPD, borderline personality disorder.

disorder may have been repeatedly let down in the past.

This does not mean that the neurologist should be expected to accept abusive or attacking behaviour (we will come on to boundary setting later). However, it can be liberating to understand that the anger or resentment manifest in front of us is unlikely to be personal but may instead result from a defensive pattern of behaviour historically adopted by the patient in the face of extreme adversity.

Such phenomena can be conceptualised by psychological theories such as projection-a proposed unconscious defence mechanism where, as a way of coping with undesirable and painful emotions, these are 'projected' onto someone else. ${ }^{14}$ In our experience, understanding of these issues often enables a calm hearing and response, which can de-escalate heated conversations and allow for more rational discourse. Such outcomes are facilitated by cultivation of one's own emotional awareness as a doctor.

\section{Knowing oneself-using one's own feelings as a clinical tool}

The doctor-patient relationship can essentially be stripped down to an interaction between two human beings, each with their own respective set of values and vulnerabilities. It is thus logical that if a patient has difficulties with emotional regulation, going from one extreme emotion to the next without obvious trigger, the encounter may provoke reactions which feel unusually and surprisingly strong in the physician. This can be referred to in psychoanalytic terms as a countertransference reaction, and, if noticed by the physician, is postulated to provide data on the patient's mental state. ${ }^{14}$ For example, a neurologist who fails to come up with a clear explanation for a patient's medical symptoms may, following an appointment with the patient, end up feeling useless and powerless. Rather than reacting defensively, the doctor may wonder whether this feeling of uselessness tells him or her something about the patient's feelings towards themselves-that is, that they are possibly connecting with the patient's own feelings of powerlessness.

\section{Maintaining appropriate boundaries}

As part of their problems in interpersonal relationships, patients with BPD sometimes have difficulty understanding and observing the boundaries of others. The issue may manifest in the form of erratic appointment attendance, excessive contact between appointments or inappropriate requests for medication or hospital admission. ${ }^{32}$ While clearly demonstrating our wish to help, it is equally important to be clear about the limits of what can be offered. It can be containing for the patient if the doctor combines empathy with consistency, transparency and clarity about appropriate doctor-patient interaction, expectations and shared management goals. ${ }^{14}$

Clear limit-setting is also encouraged in the context of pharmacological management in patients with BPD. Examples relevant to the neurologist might include tackling medication overuse for patients with headaches, ${ }^{28}$ and treading the line between collaboration and firmness when reviewing antiepileptic medication in patients with dissociative (non-epileptic) seizures. ${ }^{32}$

When setting boundaries, it can help to adopt a mentalising approach with patients, to allow them to see the rationale for the limit-setting. People with BPD have been shown to have difficulties with mentalising, which has been defined by Bateman and Fonagy as:

"the process by which we make sense of each other and ourselves, implicitly and explicitly, in terms of subjective mental processes". ${ }^{44}$ 


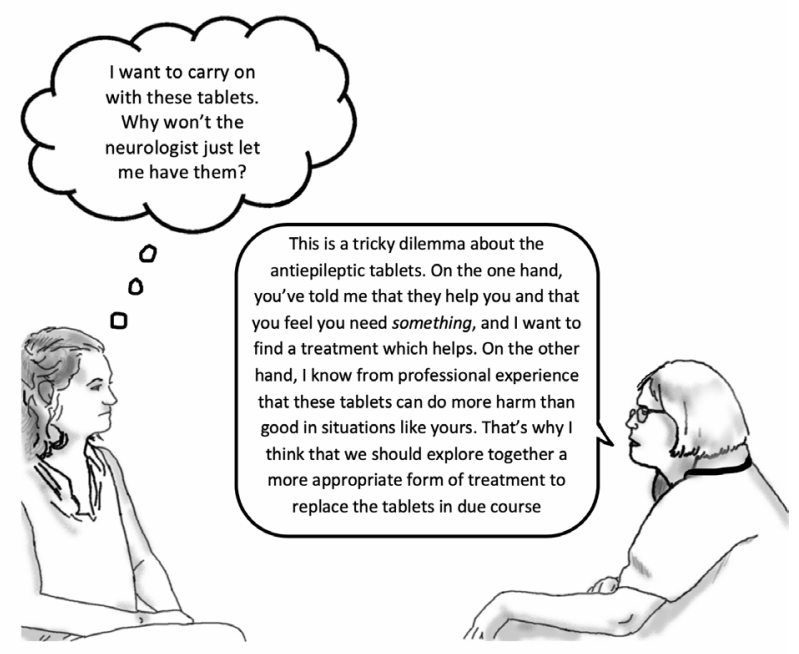

Figure 2 Suggestion on sharing a dilemma with the patient.

This can be as simple as letting the patient in on one's thought process, as exemplified in figure 2 .

\section{Responsibility and capacity}

Some clinicians are quick to characterise behaviours of people with BPD such as self-harming as 'manipulative' in nature. In our experience, the most productive approach is finding a line between encouraging patients in their agency over their health and healthcare, while not blaming them for their situation. A feeling of disempowerment can already permeate the lives of many with BPD, so the aforementioned boundaries should not be held so strictly as to add to this feeling when it comes to treatment decisions. Whenever the opportunity presents itself, encouraging a patient's sense of autonomy, competence and participation in healthcare decisions is recommended. However, in extreme situations, emotional dysregulation and the other psychological difficulties seen in BPD can sometimes cause substantial difficulties in using and weighing information. ${ }^{47}$ Thus, it is sometimes appropriate and necessary to use the Mental Capacity Act in order to enforce medical treatment in a patient's 'best interest' if there are concerns about their mental capacity in relation to treatment refusal for a medical condition. ${ }^{47}$

\section{Time and consistency}

When psychiatrists urge neurologists to take more time in consultation with people with BPD, neurologists may the there is misunderstanding of the the challenges faced in their practice. Nevertheless, Stone encourages us to reflect that neurosurgeons do not allocate the same time slots for pituitary tumour removals as for carpal tunnel decompressions. ${ }^{33}$ In a similar vein, longer initial assessments for people with BPD and complex functional neurological problems are likely to be worthwhile in the long term, as trust is built more easily with less time constraint.
For example, some patients with BPD may find aspects of the neurological examination threatening or intrusive, depending in part on previous traumatic experiences. Being particularly clear about the process and purpose of the examination, though more time-consuming, is likely to help patients to feel safe and reduce the likelihood of them misconstruing the situation.

Insofar as possible, sticking to scheduled appointments and planning follow-up is particularly pertinent in this patient group. The trust which the neurologist may have worked hard to gain is likely to need continued authentication. For patients with BPD and dissociative (non-epileptic) seizures, for example, it is advisable to continue seeing the patient at scheduled intervals (even if sparse) once the diagnosis has been made. ${ }^{32}$ This prevents the receipt of medical attention becoming conditional on escalating illness behaviours. ${ }^{32}$ Moreover, after the establishment of a therapeutic alliance, discharges should be planned and gradually phased insofar as possible. People with BPD are characteristically highly sensitive to perceived rejection which may trigger decompensation and frantic help-seeking elsewhere if this is not thoughtfully managed. ${ }^{32}$ As such, careful planning will help both the patient and the health service as a whole.

\section{Maintaining hope}

The sense of shame, anxiety and hopelessness characteristically felt by people with BPD can engender a sense of hopelessness in the treating doctor, which may itself in turn be detected by these highly sensitive patients. ${ }^{32}$ The importance of reciprocal influence within the doctor-patient relationship has been demonstrated by the finding that either more positive or negative communication on one side elicits similar

\section{Key points}

- Borderline personality disorder, frequently encountered in the general hospital setting, is associated with significant physical and psychiatric comorbidity and excess mortality.

- Borderline personality disorder is a treatable condition associated with high rates of symptom remission; however, many people experience persisting problems with personal and occupational relationships.

- Positive clinical outcomes hinge on the foundation of a therapeutic doctor-patient relationship, which presents a particular set of challenges in the context of people with borderline personality disorder.

- These challenges can often be overcome by adopting a collaborative, compassionate approach, with appropriate boundary setting.

- In parallel, maintaining a sense of hope and conveying this to the patient can have a powerful therapeutic effect. 
response styles from the other. ${ }^{48}$ Optimism and maintenance of hope on the part of the physician should therefore not be underestimated.

Moreover, this optimism is not misplaced. Though previously believed to be a treatment-resistant condition, there is now a growing body of evidence showing that patients with BPD can be effectively helped. ${ }^{44}$ As such, we believe that working with and advocating for these patients is an extremely rewarding experience.

Contributors PM and $\mathrm{KH}$ planned the outline of the paper. $\mathrm{KH}$ drafted the manuscript with input and feedback from PM.

Funding This study was supported by the NIHR Biomedical Research Centre at University Hospitals Bristol NHS Foundation Trust and the University of Bristol.

Disclaimer The views expressed in this publication are those of the authors and not necessarily those of the NHS, the National Institute for Health Research or the Department of Health and Social Care.

Competing interests None declared.

Patient consent for publication Not required.

Provenance and peer review Commissioned; externally peer reviewed by Alan Carson, Edinburgh, UK.

\section{REFERENCES}

1. National Collaborating Centre for Mental Health. Borderline personality disorder: treatment and management. National clinical practice guideline number 78. Leicester: The British Psychological Society and The Royal College of Psychiatrists, 2009.

2. Tyrer P, Reed GM, Crawford MJ. Classification, assessment, prevalence, and effect of personality disorder. Lancet 2015;385:717-26.

3. Tyrer P, Mulder R, Kim Y-R, et al. The development of the ICD-11 classification of personality disorders: an amalgam of science, pragmatism, and politics. Annu Rev Clin Psychol 2019;15:481-502.

4. American Psychiatric Association. Diagnostic and Statistical Manual of Mental Disorders. 5th ed. Washington, DC: Author, 2013.

5. Lenzenweger MF, Lane MC, Loranger AW, et al. DSM-IV personality disorders in the National Comorbidity Survey Replication. Biol Psychiatry 2007;62:553-64.

6. Trull TJ, Jahng S, Tomko RL, et al. Revised NESARC personality disorder diagnoses: gender, prevalence, and comorbidity with substance dependence disorders. J Pers Disord 2010;24:412-26.

7. Torgersen S, Kringlen E, Cramer V. The prevalence of personality disorders in a community sample. Arch Gen Psychiatry 2001;58:590-6.

8. Stepp SD, Lazarus SA, Byrd AL. A systematic review of risk factors prospectively associated with borderline personality disorder: taking stock and moving forward. Personal Disord 2016;7:316-23.

9. Grant BF, Chou SP, Goldstein RB, et al. Prevalence, correlates, disability, and comorbidity of DSM-IV borderline personality disorder: results from the Wave 2 National Epidemiologic Survey on Alcohol and Related Conditions. J Clin Psychiatry 2008;69:533-45.

10. Moran P, Jenkins R, Tylee A, et al. The prevalence of personality disorder among UK primary care attenders. Acta Psychiatr Scand 2000;102:52-7.
11. Gross R, Olfson M, Gameroff M, et al. Borderline personality disorder in primary care. Arch Intern Med 2002;162:53-60.

12. Moran P, Rendu A, Jenkins R, et al. The impact of personality disorder in UK primary care: a 1-year follow-up of attenders. Psychol Med 2001;31:1447-54.

13. Newton-Howes G, Tyrer P, Anagnostakis K, et al. The prevalence of personality disorder, its comorbidity with mental state disorders, and its clinical significance in community mental health teams. Soc Psychiatry Psychiatr Epidemiol 2010;45:453-60.

14. Linehan M. Cognitive-behavioral treatment of borderline personality disorder. New York: Guilford Press, 1993.

15. Leichsenring F, Leibing E, Kruse J, et al. Borderline personality disorder.. Lancet 2011;377:74-84.

16. Amad A, Ramoz N, Thomas P, et al. Genetics of borderline personality disorder: systematic review and proposal of an integrative model. Neurosci Biobehav Rev 2014;40:6-19.

17. Bassir Nia A, Eveleth MC, Gabbay JM, et al. Past, present, and future of genetic research in borderline personality disorder. Curr Opin Psychol 2018;21:60-8.

18. Gescher DM, Kahl KG, Hillemacher T, et al. Epigenetics in personality disorders: today's insights. Front Psychiatry 2018;9.

19. Perroud N, Salzmann A, Prada P, et al. Response to psychotherapy in borderline personality disorder and methylation status of the BDNF gene. Transl Psychiatry 2013;3:e207.

20. de Aquino Ferreira LF, Queiroz Pereira FH, Neri Benevides AML, et al. Borderline personality disorder and sexual abuse: a systematic review. Psychiatry Res 2018;262:70-7.

21. Zanarini MC, Frankenburg FR, Reich DB, et al. Biparental failure in the childhood experiences of borderline patients. $J$ Pers Disord 2000;14:264-73.

22. van Zutphen L, Siep N, Jacob GA, et al. Emotional sensitivity, emotion regulation and impulsivity in borderline personality disorder: a critical review of fMRI studies. Neurosci Biobehav Rev 2015;51:64-76.

23. Mitchell AE, Dickens GL, Picchioni MM. Facial emotion processing in borderline personality disorder: a systematic review and meta-analysis. Neuropsychol Rev 2014;24:166-84.

24. Arbabi M, Paast N, Karim HR, et al. Mild neurological impairment may indicate a psychomotor endophenotype in patients with borderline personality disorder. Psychiatry Res 2016;245:22-6.

25. Fok ML-Y, Hayes RD, Chang C-K, et al. Life expectancy at birth and all-cause mortality among people with personality disorder. J Psychosom Res 2012;73:104-7.

26. Moran P, Stewart R, Brugha T, et al. Personality disorder and cardiovascular disease: results from a National Household Survey. J Clin Psychiatry 2007;68:69-74.

27. Dixon-Gordon KL, Conkey LC, Whalen DJ. Recent advances in understanding physical health problems in personality disorders. Curr Opin Psychol 2018;21:1-5.

28. Saper JR, Lake AE. Borderline personality disorder and the chronic headache patient: review and management recommendations. Headache 2002;42:663-74.

29. Henningsen P. Management of somatic symptom disorder. Dialogues Clin Neurosci 2018;20:23-31.

30. Ludwig L, Pasman JA, Nicholson T, et al. Stressful life events and maltreatment in conversion (functional neurological) disorder: systematic review and meta-analysis of case-control studies. Lancet Psychiatry 2018;5:307-20.

31. Schmaling KB, Fales JL. The association between borderline personality disorder and somatoform disorders: a systematic 
review and meta-analysis. Clinical Psychology: Science and Practice 2018;25(2).

32. Lacey C, Cook M, Salzberg M. The neurologist, psychogenic nonepileptic seizures, and borderline personality disorder. Epilepsy Behav 2007;11:492-8.

33. Stone J. Functional neurological disorders: the neurological assessment as treatment. Pract Neurol 2016;16:7-17.

34. Jones S, Howard L, Thornicroft G. 'Diagnostic overshadowing': worse physical health care for people with mental illness. Acta Psychiatr Scand 2008;118:169-71.

35. Storebø OJ, Stoffers-Winterling JM, Völlm BA, et al. Psychological therapies for people with borderline personality disorder. Cochrane Database Syst Rev 2018;110.

36. Cristea IA, Gentili C, Cotet CD, et al. Efficacy of psychotherapies for borderline personality disorder: a systematic review and meta-analysis. JAMA Psychiatry 2017;74:319-28.

37. Stoffers JM, Völlm BA, Rücker G, et al. Psychological therapies for people with borderline personality disorder. Cochrane Database Syst Rev 2012;8.

38 National Institute for Health and Care Excellence. Borderline personality disorder: recognition and management. Clinical guideline [CG78], 2009(updated 2018). Available: https:// www.nice.org.uk/guidance/cg78/chapter/1-Guidance

39. Nock MK. Why do people hurt themselves? New insights into the nature and functions of self-injury. Curr Dir Psychol Sci 2009;18:78-83.

40. Gunderson JG, Stout RL, McGlashan TH, et al. Ten-year course of borderline personality disorder: psychopathology and function from the Collaborative Longitudinal Personality Disorders study. Arch Gen Psychiatry 2011;68:827-37.
41. Zanarini MC, Frankenburg FR, Reich DB, et al. The subsyndromal phenomenology of borderline personality disorder: a 10-year follow-up study. Am J Psychiatry 2007;164:929-35.

42. Wnuk S, McMain S, Links PS, et al. Factors related to dropout from treatment in two outpatient treatments for borderline personality disorder. J Pers Disord 2013;27:716-26.

43. Kanner AM. More controversies on the treatment of psychogenic pseudoseizures: an addendum. Epilepsy Behav 2003;4:360-4.

44. Bateman A, Campbell C, Luyten P, et al. A mentalizationbased approach to common factors in the treatment of borderline personality disorder. Curr Opin Psychol 2018;21:44-9.

45. Gunderson J. Borderline personality disorder: a clinical guide. Second Edition. American Psychiatric Publishing Inc., 2009.

46. Stone J, Hallett M, Carson A, et al. Functional disorders in the Neurology section of ICD-11: a landmark opportunity. Neurology 2014;83:2299-301.

47. Ayre K, Owen GS, Moran P. Mental capacity and borderline personality disorder. BJPsych Bull 2017;41:33-6.

48. Street RL, Gordon H, Haidet P. Physicians' communication and perceptions of patients: is it how they look, how they talk, or is it just the doctor? Soc Sci Med 2007;65:586-98.

49. Bach B, First MB. Application of the ICD-11 classification of personality disorders. BMC Psychiatry 2018;18.

50. Sansone RA, Sansone LA. Borderline personality disorder in the medical setting: suggestive behaviors, syndromes, and diagnoses. Innov Clin Neurosci 2015;12:39-44. 